\title{
Hypoglycemia in patients with congenital muscle disease
}

\author{
Leslie H. Hayes ${ }^{1,2+}$, Pomi Yun ${ }^{1 \dagger}$, Payam Mohassel ${ }^{1}$, Gina Norato ${ }^{3}$, Sandra Donkervoort ${ }^{1}$, Meganne E. Leach ${ }^{1,4}$, \\ Rachel Alvarez ${ }^{5,6}$, Anne Rutkowski, ${ }^{5,6,7}$, Natalie D. Shaw ${ }^{8}$, A. Reghan Foley ${ }^{1}$ and Carsten G. Bönnemann ${ }^{1 *}$
}

\begin{abstract}
Background: Only a few small studies have previously reported episodes of hypoglycemia in children with neuromuscular diseases; however, there has been no broader investigation into the occurrence of hypoglycemia in children with congenital muscle disease (CMD).

Methods: Pediatric patients enrolled in the CMD International Registry (CMDIR) with a history of hypoglycemia were included in this retrospective review. Hypoglycemic episodes and associated clinical and biochemical characteristics were characterized.

Results: Ten patients with CMD (5 with LAMA2-related muscular dystrophy) reported at least one episode of hypoglycemia beginning at an average age of 3.5 years. Predominant symptoms included altered mental status and nausea/vomiting, and laboratory studies demonstrated metabolic acidosis and ketonuria, consistent with ketotic hypoglycemia.

Conclusion: Patients with CMD may have an increased risk of hypoglycemia during fasting, illness, or stress due to their relatively low muscle mass and hence, paucity of gluconeogenic substrate. Clinicians should therefore maintain a high index of suspicion for hypoglycemia in this high-risk patient population and caregivers should routinely be trained to recognize and treat hypoglycemia.
\end{abstract}

Keywords: Hypoglycemia, Ketotic hypoglycemia, Congenital muscular dystrophies, Neuromuscular

\section{Background}

Hypoglycemia, defined as a blood glucose level below 60 $\mathrm{mg} / \mathrm{dl}(3.3 \mathrm{mmol} / \mathrm{L})$ in infants and children [1], has been reported sporadically in children with neuromuscular disease [2, 3]. Bruce et al. described two girls with spinal muscular atrophy type II (muscle mass only $10 \%$ of body weight) with recurrent, severe hypoglycemic episodes that went unrecognized until the girls became comatose and were found to have blood glucose levels of 30-34 $\mathrm{mg} / \mathrm{dl}(1.7-1.9 \mathrm{mmol} / \mathrm{L})$ and metabolic acidosis [2]. In one girl (case \#2), hypoglycemia frequently developed in the morning after fasting overnight [2]. Shu et al. reported a similar case; a 7-year-old boy with congenital

\footnotetext{
* Correspondence: carsten.bonnemann@nih.gov

${ }^{\dagger}$ Leslie H. Hayes and Pomi Yun contributed equally to this work.

${ }^{1}$ Neuromuscular and Neurogenetic Disorders of Childhood Section,

Neurogenetics Branch, National Institute of Neurological Disorders and

Stroke, National Institutes of Health, Building 10, Room 2B 39, MSC 1477, 10

Center Drive, Bethesda, MD 20892, USA

Full list of author information is available at the end of the article
}

muscular dystrophy with hypoglycemic episodes both upon awakening and during the course of viral illnesses [3]. A 34-h fasting study in 9 boys with Duchenne muscular dystrophy did not precipitate hypoglycemia, however these patients demonstrated lower alanine levels [4], suggesting a decreased reserve of gluconeogenic substrate from muscle. Reports of hypoglycemia are equally rare among adults with neuromuscular disease $[5,6]$. Importantly, the incidence of hypoglycemia among adults and children with neuromuscular disease has not been systematically investigated.

It has been proposed that low muscle mass in the context of muscle atrophy predisposes children with neuromuscular disorders to hypoglycemia. In healthy children, glucose levels can be maintained by hepatic glycogenolysis during periods of fasting for approximately 8-12 h [7]. As glycogen stores are depleted, however, amino acids derived from the breakdown of muscle tissue become essential substrates for hepatic gluconeogenesis [7]. In the

(c) The Author(s). 2020 Open Access This article is distributed under the terms of the Creative Commons Attribution 4.0 International License (http://creativecommons.org/licenses/by/4.0/), which permits unrestricted use, distribution, and reproduction in any medium, provided you give appropriate credit to the original author(s) and the source, provide a link to the Creative Commons license, and indicate if changes were made. The Creative Commons Public Domain Dedication waiver (http://creativecommons.org/publicdomain/zero/1.0/) applies to the data made available in this article, unless otherwise stated. 
glucose-alanine cycle, for example, alanine is released into the bloodstream by skeletal muscle and taken up by the liver [7]. There, it is converted to pyruvate which then enters the gluconeogenic pathway, creating glucose that can be shuttled back to muscle as an energy source [7]. Thus, when a patient with low muscle mass fasts or has increased energy requirements (e.g. during illness), he/she may be at risk for developing hypoglycemia due to inadequate gluconeogenic substrate from muscle. This risk is compounded in children because of their limited hepatic glycogen stores compared with adults [8, 9].

In this retrospective study, we identified 10 patients with a confirmed molecular or clinical and immunohistological diagnosis of congenital muscular dystrophy or congenital myopathy who experienced at least one episode of documented hypoglycemia. We report the frequency, common triggers, and symptoms associated with hypoglycemia episodes as well as any accompanying biochemical abnormalities available. We suggest that these patients deserve prompt evaluation of glucose levels when presenting with signs and symptoms of hypoglycemia and routine evaluation of glucose homeostasis.

\section{Methods}

An IRB-approved study announcement was distributed electronically to all members of the Congenital Muscle Disease International Registry (CMDIR) by CMDIR staff. Families who expressed interest in participating in this study were then directed to contact the investigator
(LH) via e-mail and medical information release consents were secured to request and review participants' medical records. Patients were included if they: 1) had an established clinical and molecular diagnosis of congenital muscular dystrophy or congenital myopathy (based on genetic testing and/or muscle biopsy), and 2) had a history of at least one documented episode of symptomatic hypoglycemia (defined as a blood glucose level $<60 \mathrm{mg} / \mathrm{dL}$ ) between the ages of $1-10$ years.

Of the 1702 patients registered in the CMDIR, 38 registrants had an established diagnosis of a congenital myasthenic syndrome and were not included. The remaining 1664 patients were contacted, and 17 families responded to the inquiry and were then contacted directly by the investigators. Of the 17 patients with congenital muscle disease, five did not meet inclusion/exclusion criteria because they reported isolated neonatal hypoglycemia or could not provide documentation of hypoglycemia. Two patients did not respond to further emails from the study staff. The remaining 10 patients were included in the review of records and were contacted for necessary clarifications. A flowchart of study recruitment for patients is shown in Fig. 1.

Anthropometric data was also obtained from age-, sex-, and BMI (body mass index)-matched patients with congenital muscular dystrophy without a history of hypoglycemia who were evaluated at the National Institutes of Health (NIH) as part of an IRB-approved natural history study (protocol 12-N-0095). Note that in patients

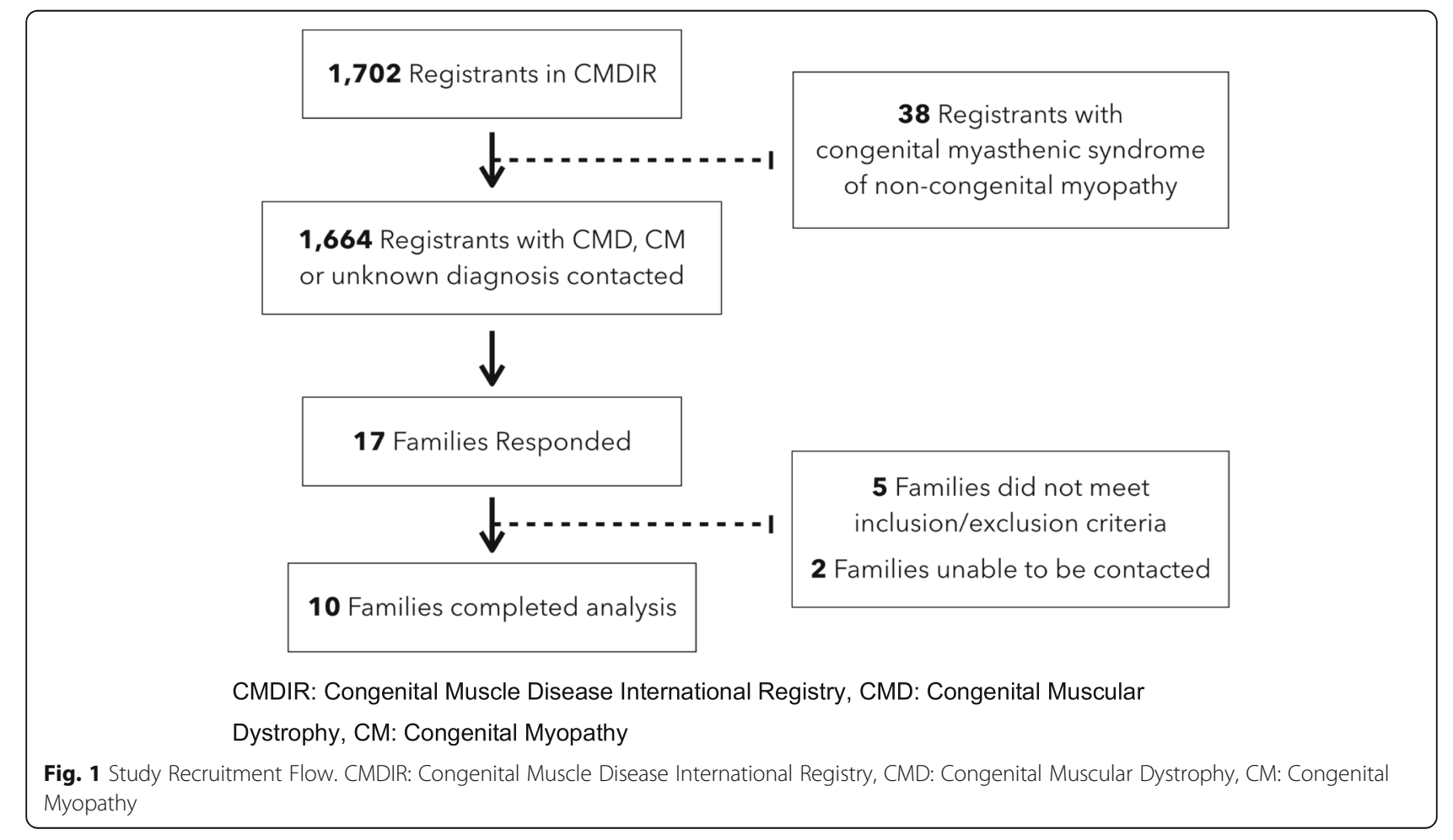


with congenital muscle disease, as well as in other patients with joint contractures, standing height is approximated using ulnar length [10].

\section{Results}

\section{Patient characteristics}

The 10 patients (6 male, 4 female) were all confirmed to have congenital muscle disease. Seven patients had congenital muscular dystrophies: LAMA2-related dystrophy $(n=5)$, COL6-related dystrophies $(n=1)$ or $\alpha$-dystroglycanopathy $(n=1)$, and three patients had congenital myopathies: DNM2-related myopathy $(n=1)$ and TTN-related myopathy $(n=2)$. Diagnoses were confirmed through review of genetic testing and/or muscle biopsy with immunohistological studies. The average age of the patient at the time of data review was 11 years. Nine out of 10 patients had additional medical records relevant to an episode of hypoglycemia, which included documentation of 32 independent hospital or emergency department visits. Additional patient characteristics are reported in Table 1.

\section{Characteristics of hypoglycemic episodes}

The first episode of confirmed hypoglycemia occurred at an average age of 3.5 years (range 1.6-7 years). While most caregivers reported eight or fewer episodes of hypoglycemia, three caregivers reported more than 15 episodes of hypoglycemia (IDs 2,3,4; Table 1). Four of the five patients who were age 10 or older at the time of the study reported complete resolution of these episodes by 10 years of age.

All patients had symptoms of lethargy/sleepiness at the time of hypoglycemic episodes. Difficulty concentrating, decreased responsiveness, and nausea/vomiting were also commonly reported symptoms (Table 2), however gastrointestinal symptoms may have been secondary to a concurrent illness rather than hypoglycemia. Of note, there were no reports of changes in vision, seizures, tremors or nightmares. Eight out of the 10 families reported general illness as a trigger for a hypoglycemic episode. Skipping meals $(n=3)$, constipation $(n=1)$ and heavy exercise $(n=1)$ were reported as additional potential triggers for hypoglycemic episodes.

\section{Medical evaluation of hypoglycemia}

Nine patients had laboratory testing and hospital records available for review. Patients were evaluated for hypoglycemia in the emergency department or during an inpatient hospital admission an average of 4 times (ranging from 1 to 7 ) per patient. Blood glucose levels ranged from 16 to $60 \mathrm{mg} / \mathrm{dl}$ (average of $45 \mathrm{mg} / \mathrm{dL}$ )), and hypoglycemia was accompanied by increased anion gap (AG) metabolic acidosis (defined as $\mathrm{pH}<7.3$, bicarbonate $<18 \mathrm{mmol} / \mathrm{L}$, and AG $>14 \mathrm{mmol} / \mathrm{L}$ ) in all cases (Fig. 2). Urine samples were collected during eight encounters and demonstrated ketonuria in $87 \%$ of samples. Patients were initially treated with normal saline (NS) (45.5\%), D5 1/2NS (22.7\%) or D10 $1 / 2 \mathrm{NS}$ (31.8\%), suggesting delayed recognition of hypoglycemia by medical providers. Indeed, medical records indicated that on at least three separate occasions, the diagnosis of hypoglycemia was not initially considered despite the presence of vomiting, decreased oral intake, and/or lethargy.

\section{Home management of hypoglycemia}

Seven families used a home glucometer to monitor blood glucose levels. Three families checked their child's blood sugar regularly regardless of symptoms (e.g. three times a day or once in the middle of the night), while the four remaining families checked blood glucose only when their child became ill, had not eaten for a prolonged period of time, or showed symptoms of hypoglycemia. Furthermore, caregivers reported frequent blood glucose levels of 60 $80 \mathrm{mg} / \mathrm{dL}$ with associated symptoms that would prompt them to intervene. In the home setting, hypoglycemia was treated with glucose-rich food $(n=6)$ or medication $(n=$ 3 ; oral glucose tablets or gel), by initiating a feed via Gtube $(n=5)$, or by using a glucagon injection kit $(n=1)$. Two additional families had been prescribed a glucagon injection kit but had never used it. No parents reported treatment of hypoglycemia with glucocorticoids. Despite having access to one or more home remedies, most families reported that their child's blood glucose did not normalize until dextrose containing IV fluids were administered.

\section{Anthropometric data}

Anthropometric data was reviewed in patients with CMD with or without a history of hypoglycemia to investigate a potential causal link between low muscle mass and hypoglycemic episodes. In the absence of available data on body composition, BMI percentile was used as a proxy for muscle mass. However, there was no difference in BMI percentiles between our cohort and patients with CMD controls who have not reported hypoglycemia. Furthermore, this cohort had BMIs that spanned a large range of percentiles, based on CDC growth charts for healthy controls (Table 1).

\section{Discussion}

In this retrospective registry-based study, we report a cohort of 10 patients with genetically and/or pathologically confirmed forms of congenital muscle disease (congenital muscular dystrophies or congenital myopathies) who experienced at least one episode of hypoglycemia. We show that these hypoglycemic episodes are typically associated with clinical symptoms, ranging from increased fatigue to decreased alertness and are often associated with profound metabolic abnormalities consistent with a ketotic hypoglycemic state. Consistent with previous reports, these 


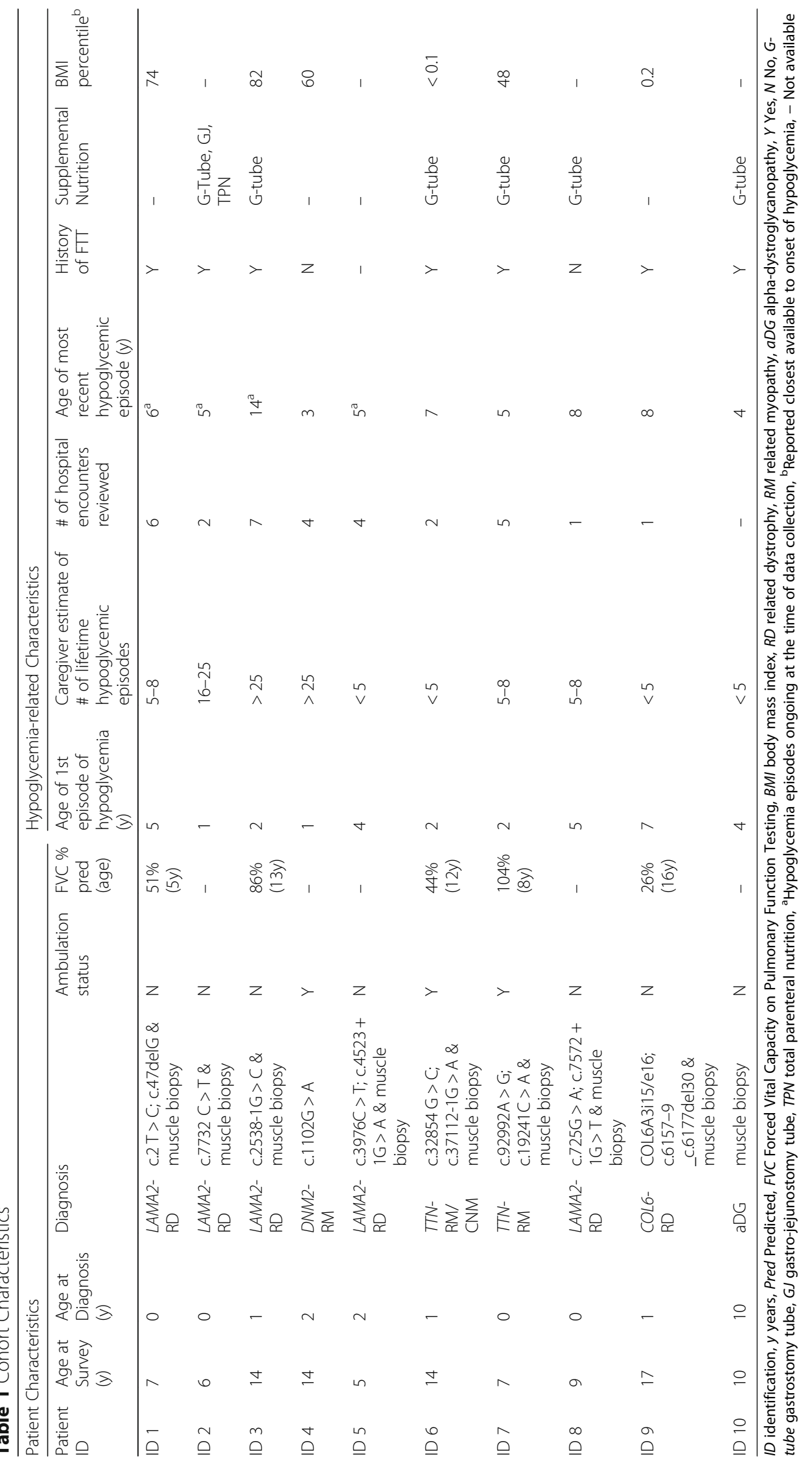


Table 2 Symptoms Reported

\begin{tabular}{ll}
\hline \# Cases & Symptoms Reported \\
\hline 10 & Lethargy/sleepiness \\
9 & Decreased responsiveness \\
& Nausea/vomiting \\
7 & Difficulty concentrating \\
6 & Loss of coordination \\
& Sweating and/or flushed \\
5 & Slurred speech \\
& Headache \\
& Fast or pounding heartbeat \\
3 & Inconsolable crying \\
& Irritability \\
& Confusion \\
& Tingling or numbness in the lips or tongue \\
& Anxiety \\
& Lightheadedness or dizziness \\
& Problems with memory \\
& Erratic behavior \\
& Nightmares \\
& Seizures \\
& Changes in vision \\
& Trembling \\
\hline &
\end{tabular}

hypoglycemic episodes are typically triggered by prolonged periods of fasting or situations of increased glucose demand such as intercurrent illness or increased physical activity. Hypoglycemic episodes were recurrent and required aggressive treatment in most of the patients studied in this cohort.

There are multiple potential mechanisms by which patients with congenital muscle disease may be at risk for developing hypoglycemia. Decreased muscle mass is believed to account for the higher risk of hypoglycemia in patients with neuromuscular disease $[5,6]$. Indeed, skeletal muscle is an essential source of amino acid substrate for gluconeogenesis during periods of prolonged fasting. While we did not have access to urine creatinine or DEXA data to estimate lean muscle mass, we did not find a strong association between hypoglycemia and BMI percentile. Further, as the majority of hypoglycemic episodes occurred in patients younger than 10 years-of-age and resolved over time, we suspect that the pathophysiology of hypoglycemia in CMD patients may overlap with that of idiopathic ketotic hypoglycemia in normal children [11].

There may also be disease-specific factors that predispose CMD patients to ketotic hypoglycemia. For example, LAMA2-related dystrophy is caused by a

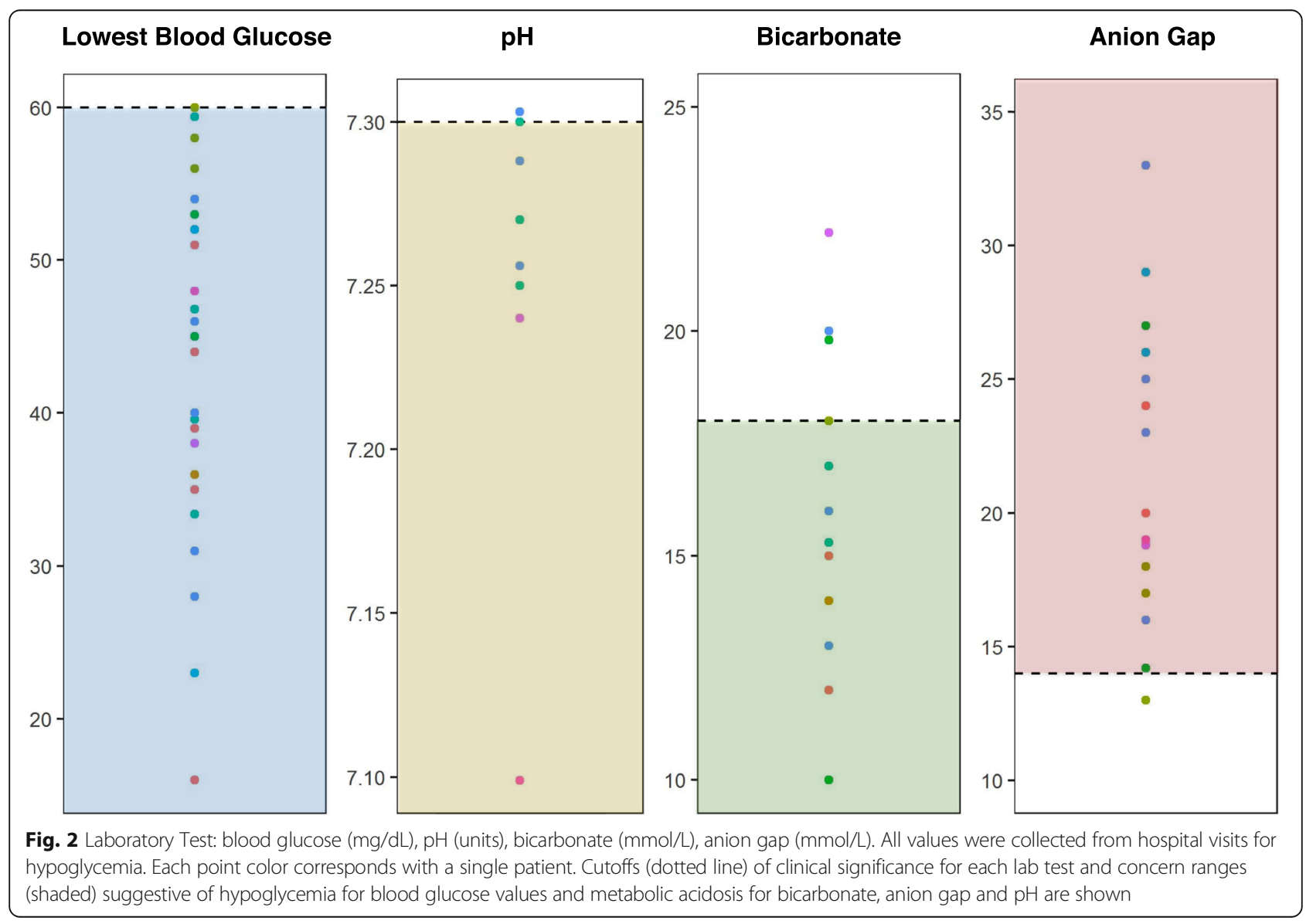


deficiency of laminin-211 (also known as merosin). It is of interest that laminin-211 is expressed not only in muscle tissue but also in the basement membrane of pancreatic insulin-producing beta cells $[12,13]$. However, hyperinsulinism is typically associated with nonketotic hypoglycemia and the majority of CMD patients had ketonuria. Another possibility is that the increased metabolic rate in pediatric patients with congenital muscle disease is contributory, as has been described in patients with Duchenne muscular dystrophy [14]. Future prospective studies in a larger patient cohort that include detailed biochemical parameters will be necessary to better understand the cause of hypoglycemia among patients with CMD.

The recruitment of patients to this study was limited by family initiative and engagement with the CMDIR and hence resulted in a low response rate. Since the small sample size and retrospective nature limits our conclusions, it will be necessary to further investigate the occurrence of hypoglycemia in patients with congenital muscle disease in a prospective study.

In conclusion, we report hypoglycemia as an important clinical occurrence in a cohort of 10 pediatric patients with congenital onset muscle disease. These patients appear to be more susceptible to hypoglycemia during early childhood, possibly due to low muscle mass resulting in inadequate gluconeogenic substrate. Thus, a glucose level should be checked in any patient who presents with an unexplained change in level of alertness, nausea and/or vomiting Specifically we recommend that (1) patients and their caregivers should monitor glucose levels during prescribed fasts (in preparation for surgery or other procedures requiring anesthesia) or during periods of illness when normal oral intake cannot be maintained; (2) first line providers should be aware of an apparent increased risk of hypoglycemic episodes in pediatric patients with congenital muscle disease and tailor diagnostic testing and fluid resuscitation protocols accordingly; and (3) providers should consider screening CMD patients for hypoglycemia with fasting glucose levels to identify those at risk.

\section{Conclusions}

Children with congenital muscle disease are at risk for episodes of ketotic hypoglycemia, often triggered by illness or fasting, which we postulate is related to low muscle mass and possible intrinsic muscle factors. Clinically, patients should be monitored closely for symptoms of hypoglycemia, be promptly evaluated and treated, and preventative measures should be considered.

\section{Abbreviations}

BMI: Body mass index; CMD: Congenital muscle disease; CMDIR: Congenital Muscle Disease International Registry; DMD: Duchenne muscular dystrophy: $\mathrm{N}$ : Number; $\mathrm{NIH}$ : National Institutes of Health; SMA: Spinal muscular atrophy

\section{Acknowledgements}

The authors thank the patients and their caregivers for participating in the study. Cure CMD, a nonprofit patient organization, and the Congenital Muscle Disease International Registry (CMDIR) assisted with patient recruitment and data collection efforts.

\section{Authors' contributions}

LHH, PM, SD, MEL, RA, AR and CGB conceived this project. LHH, PY, PM, RA and AR collected the data. LHH, PY, PM, GN and NDS analyzed the data. $L H H, P Y$ and PM drafted the manuscript, and GN, SD, MEL, RA, AR, NDS, ARF and CGB critically revised the manuscript for important intellectual content. All authors contributed to the development of the manuscript and have read and approved the final manuscript.

\section{Funding}

This work was supported, in part, by the Intramural Research Program of the $\mathrm{NIH}$, National Institute of Neurological Disorders and Stroke (CGB) and National Institute of Environmental Health Sciences (NDS), including its design, data collection, data analysis, interpretation of data and the writing of the manuscript.

\section{Availability of data and materials}

The datasets used and/or analyzed during the current study are available from the corresponding author on reasonable request.

\section{Ethics approval and consent to participate}

All patients in this retrospective study were consented to the CMDIR registry. In addition, medical information release consents were secured to obtain additional medical information pertaining to this study.

Consent for publication

Not applicable.

\section{Competing interests}

The authors declare that they have no competing interests.

\section{Author details}

${ }^{1}$ Neuromuscular and Neurogenetic Disorders of Childhood Section, Neurogenetics Branch, National Institute of Neurological Disorders and Stroke, National Institutes of Health, Building 10, Room 2B 39, MSC 1477, 10 Center Drive, Bethesda, MD 20892, USA. ${ }^{2}$ Department of Neurology, Boston Children's Hospital, 300 Longwood Ave, Boston Children's Hospital, Fegan 11, Boston, MA 02115, USA. ${ }^{3}$ Office of Biostatistics, National Institute of Neurological Disorders and Stroke, National Institutes of Health, Building 10, Room 2A 23, 10 Center Drive, Bethesda, MD 20814, USA. ${ }^{4}$ Division of Neurology, Oregon Health and Science University, 3181 SW Sam Jackson Park Rd, Portland, OR 97239, USA. ${ }^{5}$ Congenital Muscle Disease International Registry, Los Angeles, California, USA. 'C Cure CMD, 19401 S. Vermont Avenue, Suite J100, Torrance, Los Angeles, CA 90502, USA. ${ }^{7}$ Kaiser Southern California Permanente Medical Group, Los Angeles, California, USA. ${ }^{8}$ Pediatric Neuroendocrinology Group, Clinical Research Branch, National Institute of Environmental Health Sciences, Research Triangle Park, Durham, NC 27709, USA.

Received: 29 March 2019 Accepted: 3 January 2020

\section{1. -}

\section{References}

1. Thornton PS, Stanley CS, De Leon DD, et al. Recommendations from the pediatric Endocrine Society for evaluation and Management of Persistent Hypoglycemia in neonates, infants, and children. J Pediatr. 2015;167:238-45.

2. Bruce $A K$, Jacobsen $E$, Dossing $H$, et al. Hypoglycaemia in spinal muscular atrophy. Lancet. 1995;346:609-10.

3. Shu S, Cruse RP, Redmond GP. Hypoglycemia in child with congenital muscular dystrophy. Brain and Development. 1989;11:62-5.

4. Haymond MW, Strobel KE, DeVivo DC. Muscle wasting and carbohydrate homeostasis in Duchenne muscular dystrophy. Neurology. 1978;28:1224-31.

5. Builes-Montaño CE, Nieto Gonzalez M, Hernandez DL. Hypoglycemia in a patient with Facioscapulohumeral muscular dystrophy. J Clin Neuromuscul Dis. 2018;19:143-4. 
6. Orngreen MC, Zacho M, Hebert A, et al. Patients with severe muscle wasting are prone to develop hypoglycemia during fasting. Neurology. 2003;61:997-1000.

7. Melmed S, Polonsky K, Larsen PR, Kronenberg H. Williams textbook of endocrinology. 13th ed. Philadelphia: Elsevier; 2016.

8. Sunehag AL, Treuth MS, Butte NF, et al. Gluconeogenesis in children is greater than in adolescents and highly reproducible. Pediatr Res. 1998;44:450.

9. Sunehag AL, Treuth MS, Toffolo G, et al. Glucose production, gluconeogenesis, and insulin sensitivity in children and adolescents: an evaluation of their reproducibility. Pediatr Res. 2001;50:115-23.

10. Gauld LM, Kappers J, Carlin JB, et al. Height prediction from ulna length. Dev Med Child Neurol. 2004;46:475-80.

11. Chaussain JL, Georges P, Calzada L, et al. Job JC. Glycemic response to 24hour fast in normal children: III. Influence of age. J Pediatr. 1977;91:711-4.

12. Timpl R, Rohde $H$, Robey $P G$, et al. Laminin- a glycoprotein from basement membranes. J Biol Chem. 1979;254:9933-7.

13. van Deijnen $\mathrm{JH}$, Hulstaert $\mathrm{CE}$, Wolters $\mathrm{GH}$, et al. Significance of the periinsular extracellular matrix for islet isolation from the pancreas of rat, dog, pig, and man. Cell Tissue Res. 1992;267:139-46.

14. Okada K, Manabe S, Sakamoto S, et al. Protein and energy metabolism in patients with progressive muscular dystrophy. J Nutr Sci Vitaminol. 1992;38:141-54

\section{Publisher's Note}

Springer Nature remains neutral with regard to jurisdictional claims in published maps and institutional affiliations.

Ready to submit your research? Choose BMC and benefit from:

- fast, convenient online submission

- thorough peer review by experienced researchers in your field

- rapid publication on acceptance

- support for research data, including large and complex data types

- gold Open Access which fosters wider collaboration and increased citations

- maximum visibility for your research: over $100 \mathrm{M}$ website views per year

At BMC, research is always in progress.

Learn more biomedcentral.com/submissions 